\title{
Multi-sensor Fire Detection by Fusing Visual and Non-visual Flame Features
}

\author{
Steven Verstockt ${ }^{1,2}$, Alexander Vanoosthuyse ${ }^{2}$, Sofie Van Hoecke ${ }^{2}$ \\ Peter Lambert ${ }^{1}$, and Rik Van de Walle ${ }^{1}$ \\ 1 Department of Electronics and Information Systems, Multimedia Lab, \\ Ghent University - IBBT, Gaston Crommenlaan 8, bus 201, \\ B-9050 Ledeberg-Ghent, Belgium \\ 2 University College West Flanders, Ghent University Association, \\ Graaf Karel de Goedelaan 5, 8500 Kortrijk, Belgium \\ steven.verstockt@ugent.be \\ http://multimedialab.elis.ugent.be/
}

\begin{abstract}
This paper proposes a feature-based multi-sensor fire detector operating on ordinary video and long wave infrared (LWIR) thermal images. The detector automatically extracts hot objects from the thermal images by dynamic background subtraction and histogram-based segmentation. Analogously, moving objects are extracted from the ordinary video by intensity-based dynamic background subtraction. These hot and moving objects are then further analyzed using a set of flame features which focus on the distinctive geometric, temporal and spatial disorder characteristics of flame regions. By combining the probabilities of these fast retrievable visual and thermal features, we are able to detect the fire at an early stage. Experiments with video and LWIR sequences of fire and non-fire real case scenarios show good results and indicate that multi-sensor fire analysis is very promising.
\end{abstract}

\section{Introduction}

Recently, research on video analysis for fire detection has become a hot topic in computer vision. This has resulted in a large amount of vision-based detection techniques that can be used to detect the fire at an early stage 11. Based on the numerous advantages of video-based sensors, e.g. fast detection (no transport delay), indoor/outdoor detection at a distance, and the ability to provide fire progress information, it is expected that video fire detection (VFD) will become a viable alternative or complement for the more traditional fire sensors.

Although it has shown that ordinary video promises good fire detection and analysis results, we believe that the use of IR cameras in the long wave IR range (LWIR) can be of added value. Various facts corroborate this idea. First of all, existing VFD algorithms have inherent limitations, such as the need for sufficient and specific lighting conditions. Thermal IR imaging sensors image emitted light, not reflected light, and do not have this limitation, providing a 24 hour, 365 day capability. Also, the further we go in the infrared spectrum the more the visual

A. Elmoataz et al. (Eds.): ICISP 2010, LNCS 6134, pp. 333-341, 2010.

(C) Springer-Verlag Berlin Heidelberg 2010 
perceptibility decreases and the thermal perceptibility increases. As such, hot objects like flames will be best visible and less disturbed by other objects in the LWIR spectral range. Furthermore, due to the variability of shape, motion, colors, and patterns of smoke and flames, many of the existing VFD approaches are still vulnerable to false alarms. Since it is possible to integrate IR cameras into existing closed-circuit television (CCTV) networks, the combination of both technologies can be used to reduce these false alarms. At last, smoke is almost transparent in the LWIR wavelength range, and therefore, in contrast to video cameras or the human eye, a LWIR camera can look through it. As such, LWIR imaging could also be used to localize the fire through the smoke. On the basis of all these facts, the use of LWIR in combination with ordinary VFD is considered to be a win-win. This is also confirmed by our experiments, as the fused detector performs better than either sensor alone.

The remainder of this paper is organized as follows. Section 2 presents the related work in non-visible light, with a particular focus on the underlying features which can be of use in LWIR flame detection. For the related work in visible light we refer to earlier work of the authors [1. Based on the analysis of the existing approaches in visibible and non-visible light and on our experiments, Section 3 proposes the multi-sensor flame detector. Subsequently, Section 4 shows the experimental results. Section 5 ends this paper with the conclusions.

\section{Related Work in Non-visible Light}

Although the trend towards IR-based video analysis is noticeable, the number of papers about IR-based fire detection in literature is still limited. Nevertheless, the results from existing work already seem very promising and ensure the feasibility of IR video in fire detection.

Owrutsky et al. 2] work in the near infrared spectral range and focus on an increase in the global luminosity, i.e. the sum of the pixel intensities in the frame. Although this fairly simple algorithm seems to produce good results, its limited constraints do raise questions about its applicability in large and open places with varying backgrounds and a lot of ordinary moving objects. Toreyin et al. 3. detect flames in infrared by searching for bright-looking moving objects with rapid time-varying contours. A wavelet domain analysis of the $1 \mathrm{D}$-curve representation of the contours is used to detect the high frequency nature of the boundary of a fire region. In addition, the temporal behavior of the region is analyzed using a Hidden Markov Model. The combination of both temporal and spatial clues seems more appropriate than the luminosity approach and, according to the authors [3], greatly reduces false alarms. A similar combination of temporal and spatial features is used by Bosch et al. 4]. Hotspots, i.e. candidate flame regions, are detected by automatic histogram-based image thresholding. By analyzing the resulting hot objects their intensity, signature, and orientation, discrimination between flames and other hot objects is made. 


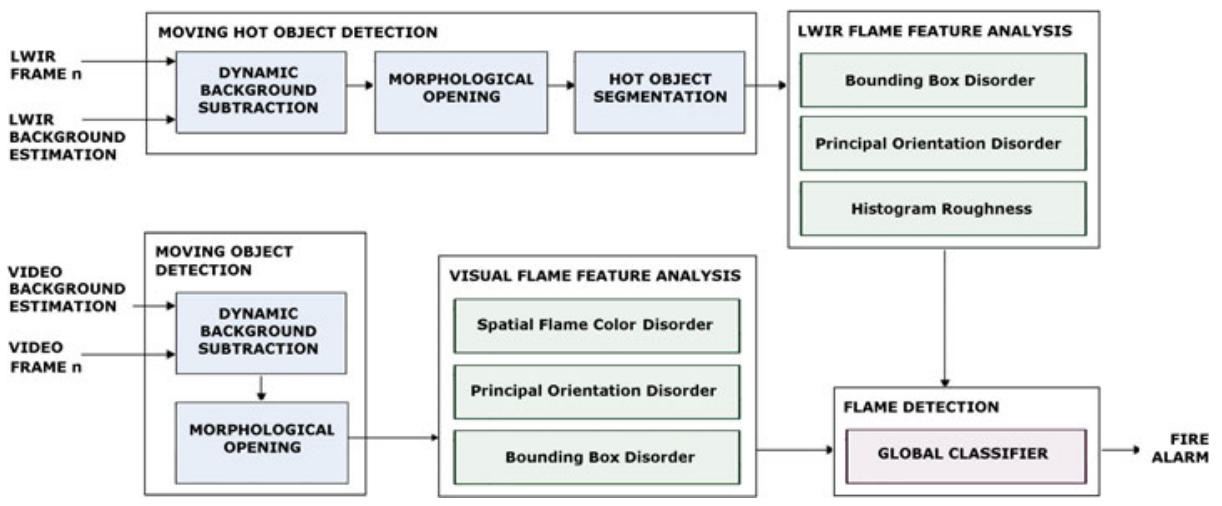

Fig. 1. General scheme of multi-sensor flame detector

\section{Multi-sensor Flame Detector}

Based on the analysis of our experiments and the related work, this section proposes the multi-sensor flame detector. As can be seen in Fig. 1, the detector consists of a video and LWIR moving (hot) object segmentation and a set of visual and LWIR flame features which analyze these objects. At the end, a global classifier combines the analysis results and takes a final decision.

\subsection{Dynamic Background Subtraction: Moving Object Detection}

Both the video and LWIR segmentation process start with a dynamic background subtraction, which extracts moving objects by subtracting the LWIR and video frames with everything in the scene that remains constant over time, i.e. the estimated background $B G_{n}$. This estimation is updated dynamically (Eq. 1) after each segmentation by comparing the pixel values of $B G_{n}$ with the values of the corresponding pixels in the frame $F_{n}$. For the LWIR images the comparison is based on the temperature increase of flames: if the quantized shifted value of the pixel $[x, y]$ in $F_{n}$ is higher than the shifted value of the pixel in $B G_{n}, F_{n}[x, y]$ is labeled as foreground $F G$. Otherwise, $F_{n}[x, y]$ gets a background label $B G$. The shifting is performed by moving down the value of each pixel in $B G_{n}$ to the nearest ten and by shifting the corresponding pixel in $F_{n}$ over the same distance. The quantization is done by rounding off the shifted values of $F_{n}$ to the nearest ten. For the ordinary video, a similar approach is used. $F_{n}[x, y]$ is labeled as $F G$ if the quantized shifted intensities of the pixel in $B G_{n}$ and $F_{n}$ differ.

$$
B G_{n+1}[x, y]= \begin{cases}\alpha B G_{n}[x, y]+(1-\alpha) F_{n}[x, y] & \text { if } F_{n}[x, y] \rightarrow B G \\ B G_{n}[x, y] & \text { if } F_{n}[x, y] \rightarrow F G\end{cases}
$$

where the update parameter $\alpha$ is a time constant that specifies how fast new information supplants old observations. Here $\alpha(=0.95)$ was chosen close to 1 . 


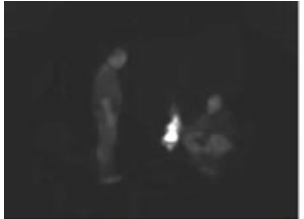

LWIR-VIDEO frame n

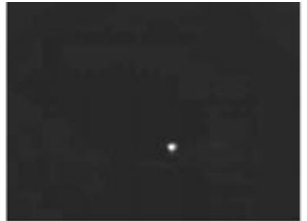

BACKGROUND ESTIMATION

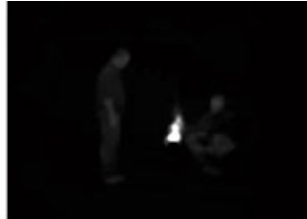

BACKGROUND SUBTRACTION

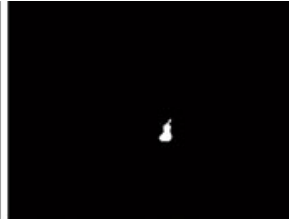

HOT OBJECT SEGMENTATION

Fig. 2. Hot object segmentation by background subtraction and histogram-based dynamic thresholding

To avoid unnecessary computational work and to decrease the number of false alarms caused by noisy objects, a morphological opening, with a $3^{*} 3$ square structuring element, is performed after the dynamic background subtraction. Each of the remaining FG objects in the video images is then further analyzed using a set of visual flame features. Analogously, LWIR FG objects are analyzed by a set of LWIR flame features. Before this LWIR analysis starts, a histogram-based segmentation is used in addition to extract the hottest objects out of the set of LWIR FG objects. Only these hot FG objects are further analyzed.

\subsection{LWIR Hot Object Segmentation}

Like in the work of Bosch et al. 4] we extract the hot objects representing possible flames by separating the highly brightened objects from the less brightened objects (Fig. 2). This segmentation step uses Otsu's method [5], which automatically performs histogram shape-based image thresholding, assuming that the image to be processed contains two classes of objects. Iteratively the optimum threshold separating those two classes is calculated so that their combined spread, i.e. the intra-class variance (Eq. 2), is minimal.

$$
\sigma_{w}^{2}(t)=\omega_{1}(t) \sigma_{1}^{2}(t)+\omega_{2}(t) \sigma_{2}^{2}(t)
$$

where weights $\omega_{i}$ are the probabilities of the two classes separated by a threshold $t$ and $\sigma_{i}^{2}$ are the variances of these classes.

\subsection{Hot Object Analysis: LWIR Flame Features}

Bounding Box Disorder (BBD): Our experiments (Fig. 3) revealed that the bounding box $\mathrm{BB}$ of flames varies considerably over time in both directions and that this variation shows a high degree of disorder. As such, the $B B D$ (Eq. 3) is chosen as a feature to distinguish between flames and other hot objects. It is related to the number of extremes, i.e., local maxima and minima, in the set of $N B B_{\text {width }}$ and $B B_{\text {height }}$ data points. By smoothing these data points, small 

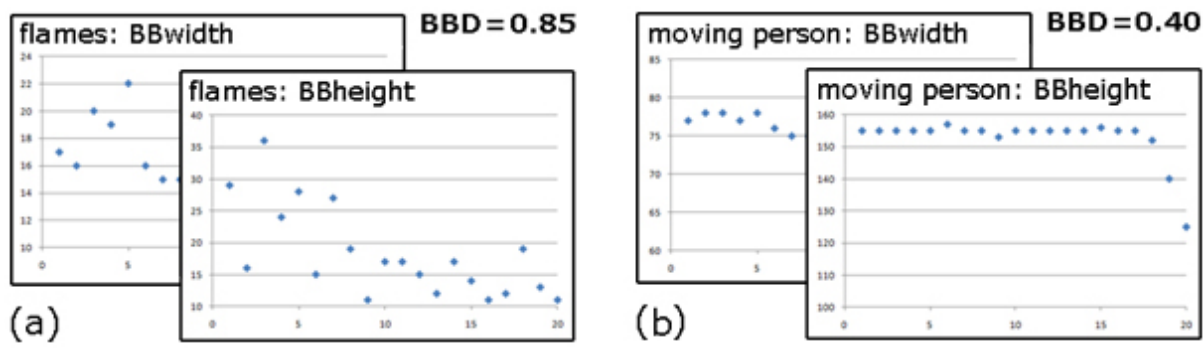

Fig. 3. Bounding box disorder of flames (a) and moving person (b)

differences between consecutive points are filtered out and are not taken into account in the extrema calculation, which increases the strength of the feature. Flames, with a high number of extremes, will have a $B B D$ close to 1 , while for more static objects it will be near to 0 .

$$
B B D=\frac{\mid \text { extrema }\left(B B_{\text {width }}\right)|+| \text { extrema }\left(B B_{\text {height }}\right) \mid}{N}
$$

Principal Orientation Disorder (POD): During the experiments, it was also found that the disorder in principal orientation is remarkably higher for flames than for more static objects like people. This orientation equals the angle $\alpha$ between the x-axis and the major axis of the ellipse that has the same secondmoments as the object region. The POD focuses on this orientation disorder characteristic and is calculated in a similar way as the BBD (Eq. 4).

$$
P O D=\frac{|\operatorname{extrema}(\alpha)|}{N / 2}
$$

Again, flames, with a high number of orientation extremes, will have a POD close to 1 , while more static objects their POD will be near to 0 .

Histogram Roughness (HR): By inspection of the histograms $\mathrm{H}$ of hot objects, it was observed that histograms of flame regions are very rough (Fig. 4). Also, it was found that the intensities of these regions range almost over the whole histogram, while for non-flame objects these intensities are more centered on some specific intensity bins and have a smaller range. The $H R$ focuses on these two findings. As Eq. 5 shows, the $H R$ equals the mean range of the histogram multiplied by the average disorder over all the non-zero bins. This average disorder is also calculated by extrema analysis and is the indicator of the histogram roughness over time.

$$
H R=\frac{\overline{\text { range }(H)}}{N} * \frac{\overline{\mid \text { extrema }_{\text {bins } \neq \emptyset}(H) \mid}}{N / 2}
$$




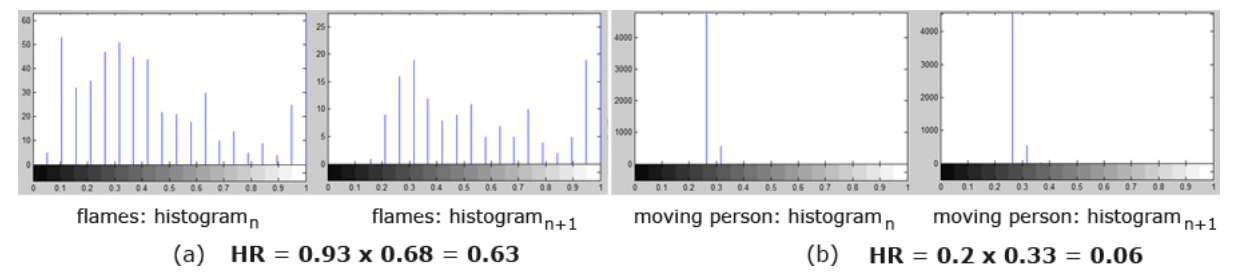

Fig. 4. Histogram analysis of flames (a) and moving person (b)

\subsection{Moving Object Analysis: Visual Flame Features}

The set of visual flame features, to analyze moving objects in ordinary video, also consists of three features. Two of them, i.e. BBD and POD, are equal to their LWIR equivalents. The other feature is the spatial flame color disorder, which focuses on the color characteristics of the object.

Spatial Flame Color Disorder (SFCD): Based on our experiments and the work of others [6], it is reasonable to assume that the colors of flames belong to the red-yellow color range. Furthermore, experiments showed that the flame color does not remain steady, i.e. flames are composed of several varying colors. The SFCD focuses on both color-related aspects of flames in order to eliminate non fire-colored objects and ordinary fire-colored objects with a solid flame color. As is defined in Eq. 6, the $S F C D$ is calculated as the product of the percentage flame pixels and the spatial disorder in the object region $R$. The percentage flame pixels equals the ratio of the number of pixels $\#_{Y-R}\left(R_{H}\right)$ with a hue value in the yellow-red range and the total number of pixels $\#_{\text {pixels }}(R)$. The spatial disorder equals the ratio of the average range over all sets $\Omega_{I}([x, y])$ of 4-neighboring pixels' intensities and the overall range of the pixels' intensities in the object region. Objects with a SFCD close to 1 will most probably represent flames, while ordinary moving objects will have a SFCD close to 0.

$$
\begin{aligned}
\Omega([x, y]) & =\{[x, y-1],[x-1, y],[x, y],[x+1, y],[x, y+1]\} \\
S F C D & =\frac{\#_{Y-R}\left(R_{H}\right)}{\#_{\text {pixels }}(R)} * \frac{\frac{\operatorname{range}\left(\Omega_{I}([x, y])\right)}{\operatorname{range}\left(R_{I}\right)+\varepsilon}}{}
\end{aligned}
$$

\subsection{Global Classifier}

Each of the proposed visual and LWIR flame features possesses a probability between 0 and 1, indicating whether the object has the flame characteristic. By averaging these LWIR and visual flame probabilities (Eq. 7), the probabilities $P_{L W I R}\left(\right.$ Flames) and $P_{\text {video }}($ Flames) are retrieved, which indicate wether the object should be classified as flames in the respective spectral range. The global classifier combines these two probabilities, using (Eq. 8), into an overall flame 
probability $P($ Flames $)$. The parameter $\beta$ in this equation is a constant that specifies how much of $P_{L W I R}\left(\right.$ Flames) and $P_{\text {video }}$ (Flames) must be taken into account in the overall probability calculation. Depending the circumstances, e.g. night or day, an appropriate $\beta$ value can be chosen. At the end, the overall probability $P$ (Flames), is compared to an alarm threshold $t_{\text {fire }}$. If the flame probability is high enough, a fire alarm is raised. In our experiments it was found that a good value for $t_{\text {fire }}$ is 0.7 .

$$
\begin{gathered}
P_{L W I R}(\text { Flames })=\frac{B B D+P O D+H R}{3} \\
P_{\text {video }}(\text { Flames })=\frac{B B D+P O D+S F C D}{3} \\
P(\text { Flames })=\beta * P_{L W I R}(\text { Flames })+(1-\beta) * P_{\text {video }}(\text { Flames })
\end{gathered}
$$

\section{Experimental Results}

The proposed multi-sensor flame detector was tested with a Xenics Gobi-384 LWIR camera [7] and a Linksys WVC2300 camera, which works in the $8-14$ $\mu \mathrm{m}$ spectral range and the visible spectrum respectively. Using the Xenics Xeneth software we were able to extract appropriate grayscale video images out of the thermal imaging camera. These images were then further analyzed by our own LWIR detection algorithm written in Matlab, extended with some add-ons for extrema detection and histogram analysis. The images from the Linksys camera, which were already in the appropriate MPEG-4 video format, were analyzed by the video-based flame detector, also written in Matlab. At the end, the probabilities of both detections were combined, using the global classifier (Eq. 8, $\beta=0.6$ ), and a final indication about the presence of flames is given.

The video and LWIR images in Fig. 5 are some exemplary frames of the fire and non-fire realistic video sequences, which were captured to test the proposed multi-sensor flame detection algorithm. Table 1 summarizes the detection results for all the tested sequences. As the results in the Table indicate, the proposed algorithm yields good detection results. For uncontrolled fires, e.g. burning paper, the flame detection rate is higher than $90 \%$ and for controlled fires, e.g. a bunsen burner, it is around $75 \%$. Furthermore, the number of false detections is very low. By further analyzing the detection results, e.g. by temporal median filtering, it is expected that this number of false alarms can even be further reduced. However, to confirm all these findings a more thorough evaluation and comparison with other work [2-4] is needed. This will be part of our future work.

Changing the $\beta$ value in Eq. 8 to 0 or 1 , transforms the multi-sensor detector into a standalone LWIR or video fire detector respectively. Comparisson of the results for these standalone detectors with the results of our multi-sensor approach have confirmed our hypothesis that the fused detector performs remarkably better than either sensor alone. 
Table 1. Performance evaluation of multi-sensor flame detector

\begin{tabular}{lccccc}
\hline $\begin{array}{l}\text { Video sequence } \\
\text { \# frames) }\end{array}$ & $\begin{array}{c}\text { \# fire } \\
\text { frames }\end{array}$ & $\begin{array}{c}\text { \# detected } \\
\text { fire frames }\end{array}$ & $\begin{array}{c}\text { mean } \\
\text { P(flames) }\end{array}$ & $\begin{array}{c}\text { \# false } \\
\text { detections }\end{array}$ & $\begin{array}{c}\text { flame } \\
\text { detection } \\
\text { rate }\end{array}$ \\
\hline \hline $\begin{array}{l}\text { Attic1 (337) } \\
\text { Fire } \rightarrow \text { burning paper }\end{array}$ & 264 & 259 & 0.92 & 6 & 0.96 \\
$\begin{array}{l}\text { Attic2 (2123) } \\
\text { Fire / Moving people }\end{array}$ & 1461 & 1352 & 0.84 & 19 & 0.91 \\
$\begin{array}{l}\text { Attic3 (886) } \\
\text { Moving people }\end{array}$ & 0 & 5 & 0.22 & 5 & - \\
$\begin{array}{l}\text { Lab (115) } \\
\text { Fire } \rightarrow \text { bunsen burner }\end{array}$ & 98 & 74 & 0.77 & 0 & 0.75 \\
$\begin{array}{l}\text { Corridor1 (622) } \\
\text { Moving people }\end{array}$ & 0 & 0 & 0.19 & 0 & - \\
$\begin{array}{l}\text { Corridor2 (184) } \\
\text { Moving people / Hot object }\end{array}$ & 0 & 3 & 0.28 & 3 & - \\
\hline
\end{tabular}

* detection rate $=$ (\# detected fire frames - \# false alarms) / \# fire frames
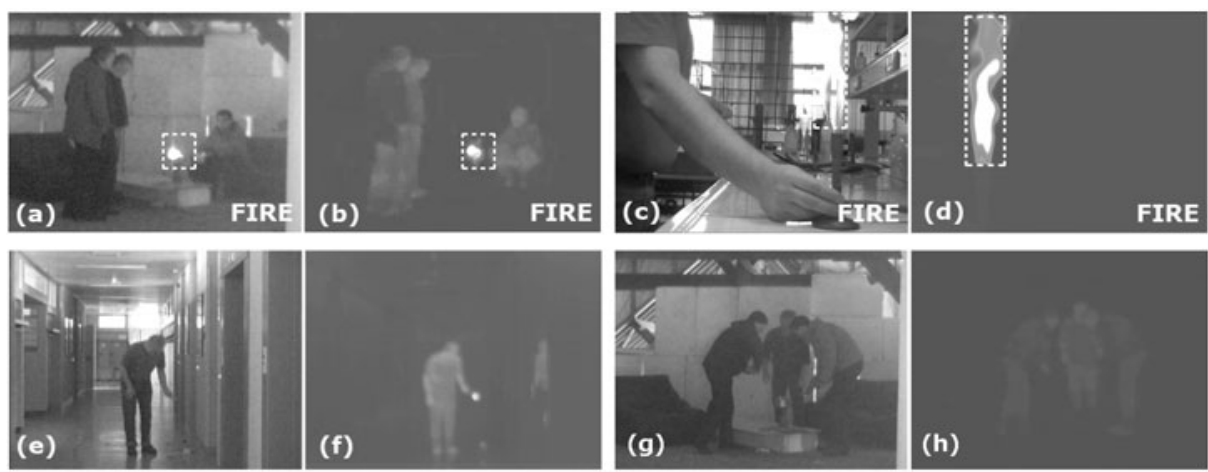

Fig. 5. Test sequences in ordinary video (a,c,e,g) and LWIR (b,d,f,h): Attic2, Lab, Corridor2, and Attic3

\section{Conclusions}

To detect the presence of fire at an early stage, the proposed multi-sensor fire detector fuses visual and non-visual flame features from moving (hot) objects in ordinary video and long wave infrared (LWIR) thermal images. Extraction of the moving objects is based on dynamic background subtraction. Additionally, LWIR moving objects are further filtered by histogram-based hot object segmentation, such that only the hottest moving objects remain. These hot and moving objects are then further analyzed using a set of flame features which focus on 
the distinctive geometric, temporal and spatial disorder characteristics of flame regions. By combining the probabilities of the bounding box disorder (BBD), the principal orientation disorder (POD), and the histogram roughness of hot moving objects in LWIR, a LWIR flame probability is calculated. Analogously, the probabilities of the spatial flame color disorder, the BBD, and the POD of moving objects in ordinary video are combined into a video flame probability. At the end, both the LWIR and the video flame probability are combined into a multi-sensor flame probability, which gives an indication about the presence of flames. If this indication is high enough, a fire alarm is given.

Experimental results have shown that the proposed multi-sensor flame detector already yields good results, but further testing on a broader range of video sequences and comparisson with existing work is necessary for a more adequate performance evaluation. At the moment, however, we can already say that multi-sensor fire analysis is very promising.

Acknowledgements. The research activities as described in this paper were funded by Ghent University, the Interdisciplinary Institute for Broadband Technology (IBBT), University College West Flanders, the Institute for the Promotion of Innovation by Science and Technology in Flanders (IWT), the Fund for Scientific Research-Flanders (FWO-Flanders), the Belgian Federal Science Policy Office (BFSPO), and the European Union.

\section{References}

1. Verstockt, S., Merci, B., Sette, B., Lambert, P., Van de Walle, R.: State of the art in vision-based fire and smoke detection. In: Proc. 14th International Conference on Automatic Fire Detection, pp. 285-292 (2009)

2. Owrutsky, J.C., Steinhurst, D.A., Minor, C.P., Rose-Pehrsson, S.L., Williams, F.W., Gottuk, D.T.: Long Wavelength Video Detection of Fire in Ship Compartments. Fire Safety Journal 41, 315-320 (2006)

3. Toreyin, B.U., Cinbis, R.G., Dedeoglu, Y., Cetin, A.E.: Fire Detection in Infrared Video Using Wavelet Analysis. SPIE Optical Engineering 46, 1-9 (2007) 067204

4. Bosch, I., Gomez, S., Molina, R.: Object discrimination by infrared image processing. In: Mira, J., Ferrández, J.M., Álvarez, J.R., de la Paz, F., Toledo, F.J. (eds.) IWINAC 2009. LNCS, vol. 5602, pp. 30-40. Springer, Heidelberg (2009)

5. Otsu, N.: A threshold selection method from gray-level histograms. IEEE Transactions on Systems, Man and Cybernetics 9, 62-66 (1979)

6. Chen, T.-H., Wu, P.-H., Chiou, Y.-C.: An early fire-detection method based on image processing. In: Proc. International Conference on Image Processing, pp. 1707-1710 (2004)

7. Xenics Infrared Solutions: Situational Firefighting, http://www.xenics.com/ 